\title{
Prefermentation improves ethanol yield in separate hydrolysis and cofermentation of steam-pretreated wheat straw
}

\author{
Fredrik Nielsen, Guido Zacchi, Mats Galbe and Ola Wallberg* (1)
}

\begin{abstract}
Agricultural residues, such as wheat straw, are feasible substrates for ethanol fermentation provided that pentoses and hexoses can be converted efficiently. Separate hydrolysis and cofermentation (SHCF) constitute a framework for improvement of conversion efficiency, because it permits independent optimization of the enzymatic hydrolysis and cofermentation steps. A drawback is that the high glucose concentrations present in SHCF repress xylose utilization and constrain ethanol yields. To improve xylose utilization the xylose-rich hydrolyzate liquor was separated from glucose-rich solids and the phases were cofermented sequentially. Prefermentation of the xylose-rich hydrolyzate liquor followed by fed-batch cofermentation of glucose-rich prehydrolyzed solids enabled sequential targeting of xylose and glucose conversion. The aim was to improve the xylose conversion by lowering the glucose repression of the xylose uptake. Various prefermentation configurations and feed patterns for prehydrolyzed solids were examined. Prefermentation increased ethanol yields overall, and fed-batch prefermentation reduced xylitol production. The best results were obtained by balancing promotion of efficient xylose conversion with maintained yeast viability. Fedbatch prefermentation and a single addition of prehydrolyzed solids, elicited an ethanol yield of $0.423 \mathrm{~g} \cdot \mathrm{g}^{-1}$ and a xylitol yield of $0.036 \mathrm{~g} \cdot \mathrm{g}^{-1}$.
\end{abstract}

Keywords: Saccharomyces Cerevisiae, Xylose, Cofermentation, Prefermentation, Prehydrolysis, Lignocellulose, Ethanol

\section{Background}

Fermentative conversion of lignocellulosic biomass into ethanol provides a sustainable alternative that could partially replace traditional petroleum refining, but to successfully implement lignocellulosic technologies economic sustainability must be ensured. High final ethanol concentration and high ethanol yield has been identified as key factors for improved process economics [1]. To achieve these, efficient hydrolysis and fermentation as well as utilization of a variety of sugars present in the feedstock are necessary.

The main obstacles to efficient cofermentation of lignocellulose-derived sugars are the limitations of the microbial physiology that restricts efficient conversion of various substrates [2] and the ability to cope with a

\footnotetext{
${ }^{*}$ Correspondence: ola.wallberg@chemeng.lth.se Department of Chemical Engineering, Lund University, P.O. Box 124, 22100 Lund, Sweden
}

variety of inhibitors [3]. The wild-type strain of Saccharomyces cerevisiae is tolerant to many inhibitors that are generated by thermochemical pretreatment, but it is largely unable to convert pentoses into ethanol without genetic modification [4]. Exogenous genes that encode for xylose reductase (XR) and xylitol dehydrogenase $(\mathrm{XDH})[5,6]$, as well as xylose isomerase [7], have been introduced into the $S$. cerevisiae genome to enable assimilation of xylose. However, fermentation of xylose to ethanol by engineered S. cerevisiae is slower and generally results in lower ethanol yields than glucose fermentation [8]. This is likely because of limitations in capacity in the pentose phosphate shunt [9] and an imbalance in redox cofactors in engineered $\mathrm{XR} / \mathrm{XDH}$-pathways [10]. The cofactor imbalance between the NAD $(\mathrm{P}) \mathrm{H}$-consuming $\mathrm{XR}$ and $\mathrm{NADH}$-producing $\mathrm{XDH}$ catalyzed reactions restricts flux through the engineered pathway, and causes xylitol production [11, 12]. Improvements have been 
made by altering the cofactor specificity of XR towards NADH $[13,14]$, and by overexpression of endogenous xylulokinase [9]. However, the distribution of products and the rate of conversion remain dependent on the balance and turnover of cofactors. The xylose conversion capacity, as well as tolerance of inhibitors, have been further improved in engineered S. cerevisiae strains by evolutionary engineering and adaptation strategies [15], but the slow cellular uptake of pentoses remains a constraint for efficient xylose conversion. Xylose is taken up by nonspecific hexose transport mechanisms [16], and because their affinity for glucose is many-fold higher than for xylose [10] excessive amounts of glucose competitively inhibit the transporters and prevent efficient uptake of xylose. However, low concentrations of glucose have been shown to enhance the xylose uptake rate [17], which imply coconsumption [18]. The enhanced xylose uptake rate has been attributed to improved cofactor recycling [19], and the induction of genes expression for transporter systems [19] and glycolytic enzymes [20].

Fermentation design can provide a tool to improve xylose utilization and conversion efficiency by accommodating the substrate consumption patterns of the fermenting microorganism. Thus, various strategies have been proposed to optimize the conversion of biomassderived glucose and xylose to ethanol, where enzymatic hydrolysis and fermentative conversion can be performed either sequentially (separate hydrolysis and cofermentation, or SHCF) or simultaneously in a single vessel (simultaneous saccharification and cofermentation, or SSCF). Opting for either strategy is generally a trade-off between optimal temperatures and inhibitory glucose concentrations during hydrolysis on the one hand (SHCF) and suboptimal temperatures and ethanol-inhibited cellulolysis on the other (SSCF). Whereas some studies have shown that SSCF-based designs generally result in higher yields $[18,21]$, the separate hydrolysis in SHCF-based designs enable optimization of the process conditions in the individual steps. Performing separate hydrolysis eliminates rate limiting effects of the hydrolysis on conversion rates, and problems associated with high viscosity during fermentation are alleviated by prior liquefaction of the solids. These properties become increasingly important as the solids load is increased in the process. Both strategies have advantages, and the choice is strain and feedstock dependent. Modifications to the fundamental strategies have been implemented to improve fermentation performance and substrate utilization [22-24]. Fed-batch design has been implemented to promote coconsumption in SHCF $[22,23]$. Fed-batch designs, where a glucose-rich feed supported the xylose utilization, improved the overall ethanol yields, and lowered xylitol production in co-fermentation of steam-pretreated wheat straw with strains of xylose-fermenting S. cerevisiae [23]. Further, prefermentation has been implemented as a modification to SSCF to improve xylose utilization and ethanol yields [24, 25]. Depletion of glucose in the liquid fraction of whole spruce slurry, prior to enzyme addition in SSCF, reduced the competitive inhibition of the xylose uptake and increased ethanol yields [24]. The authors presupposed that the process significance would be even greater with xylose-rich feedstocks. When pretreated agricultural residues are used as substrate, which have higher xylose content than spruce, the use of prefermentation can be extended to encompass substantial xylose conversion. The high xylose concentration, in combination with low glucose concentration, in the hydrolyzate liquor provide glucose-to xylose ratios during prefermentation that kinetically favor xylose uptake [17]. By separating the hydrolyzate liquor from the lignocellulosic solids, the advantages of an SHCF strategy can be combined with the beneficial conditions for xylose conversion in prefermentation. The combined strategy features sequential targeting of xylose and glucose conversion with optimal temperatures and customization of the enzymatic hydrolysis and fermentation steps individually. It has previously been demonstrated with a 2-step batch-SSCF of AFEX-pretreated switchgrass that sequential targeting of xylose and glucose conversion improve xylose utilization and ethanol yields [25].

In this study, various SHCF-based cofermentation strategies for the conversion of glucose and xylose to ethanol were examined. Two wheat straw slurries with various inhibitor concentrations, prepared by dilute acid catalyzed steam-explosion, were used. The pentose-rich hydrolyzate liquor in the slurries was separated from the hexose-rich solids to enable sequential targeting of xylose and glucose conversion. Cofermentation was performed by a xylose-fermenting and inhibitor tolerant strain of $S$. cerevisiae in a 2-step process, where prefermentation of the hydrolyzate liquor was followed by feeding of enzymatic hydrolysate, which consisted of prehydrolyzed unwashed solids. The hypothesis was that xylose could be converted with greater efficiency and render higher ethanol yields under the more favorable conditions for xylose conversion in the sequential fermentation steps than by conventional SHCF. Various feed patterns during prefermentation and for the addition of enzymatic hydrolyzate were examined to improve the xylose conversion and maximize the ethanol yield.

\section{Methods}

\section{Microorganisms}

Fermentation was performed with the non-commercial recombinant Saccharomyces cerevisiae KE6-12 strain (Taurus Energy AB), which harbors genes from 
Scheffersomyces stipitis (formerly Pichia stipitis) that encode for xylose reductase (XR) and xylitol dehydrogenase $(\mathrm{XDH})$ and overexpressing endogenous xylulokinase (XK), thus enabling xylose conversion. The strain was developed by evolutionary engineering [26] on the industrial strain S. cerevisiae TMB3400 [27] to improve inhibitor tolerance and xylose conversion capacity. Stock culture aliquots contained a mass fraction of $20 \%$ glycerol in water and were stored at $-80^{\circ} \mathrm{C}$.

\section{Raw material and preprocessing}

Two batches of wheat straw that were pretreated with dilute acid-catalyzed steam explosion under various conditions were procured from SEKAB E-Technology $A B$ (Örnsköldsvik, Sweden). The slurries were denoted severe or mild slurry, respectively, based on the relative inhibitor concentrations in the hydrolyzate liquor. The severe slurry was prepared by impregnation with dilute $\mathrm{H}_{2} \mathrm{SO}_{4}$, $\mathrm{pH}$ 2.4, and steam-pretreatment at $190{ }^{\circ} \mathrm{C}$ for $15 \mathrm{~min}$, and had a water-insoluble solids (WIS) content of 13.9 wt \% and a total dry-matter (DM) content of $20.1 \mathrm{wt} \%$. The mild slurry was prepared by impregnation of wheat straw with dilute $\mathrm{H}_{2} \mathrm{SO}_{4}, \mathrm{pH} 1.7$, and steam-pretreatment at $187{ }^{\circ} \mathrm{C}$ for $8 \mathrm{~min}$, and had a WIS content in the range of 11.1-12.7 wt \% and a total DM content in the range of 17.5-18.2 wt \%. The pH of the slurries was adjusted to 5 with $12.5 \mathrm{M} \mathrm{NaOH}$, and the hydrolyzate liquor was separated from the solids by filtration using a hydraulic press (HP5 M, Fischer Maschinenfabrik). The unwashed solid fraction of the severe slurry had a WIS content of $34 \mathrm{wt} \%$ after filtration, versus between 38 and 44 wt \% WIS in the unwashed solid fraction of the mild slurry.

\section{Cultivation of yeast}

The precultures were cultivated in $250 \mathrm{ml}$ shake flasks with $150 \mathrm{ml}$ of sterile minimal medium, containing $20 \mathrm{~g} \cdot \mathrm{L}^{-1}$ glucose, $20 \mathrm{~g} \cdot \mathrm{L}^{-1}$ xylose, $7.5 \mathrm{~g} \cdot \mathrm{L}^{-1}\left(\mathrm{NH}_{4}\right)_{2} \mathrm{SO}_{4}$, $3.75 \mathrm{~g} \cdot \mathrm{L}^{-1} \mathrm{KH}_{2} \mathrm{PO}_{4}$, and $0.75 \mathrm{~g} \cdot \mathrm{L}^{-1} \mathrm{MgSO}_{4} 7 \mathrm{H}_{2} \mathrm{O}$. The media was supplemented with $1 \mathrm{~mL} \cdot \mathrm{L}^{-1}$ vitamin solution and $10 \mathrm{~mL} \cdot \mathrm{L}^{-1}$ trace element solution, per Taherzadeh et al. [28]. The $\mathrm{pH}$ of the medium was adjusted to 5.5 with $5 \mathrm{M} \mathrm{NaOH}$ in all precultures, all of which were inoculated with $300 \mu \mathrm{l}$ of the stock cell aliquots. The preculture was incubated at $30^{\circ} \mathrm{C}$ on an orbital shaker (LabTherm, Kühner) at $180 \mathrm{rpm}$ for $24 \mathrm{~h}$.

The cultivations were performed in a sterilized $2 \mathrm{~L}$ Labfors bioreactor (Infors AG) equipped with two six-blade Rushton turbines. The reactor diameter to impeller diameter ratio was 3 , and the reactor height to diameter ratio was 1.7. The yeast was propagated with aerobic batch cultivation on molasses followed by aerobic fed-batch cultivation on wheat straw hydrolyzate liquor and molasses. The batch cultivation was performed with $50 \mathrm{~g} \cdot \mathrm{L}^{-1}$ molasses solution that was supplemented with $23.5 \mathrm{~g} \cdot \mathrm{L}^{-1}$ $\left(\mathrm{NH}_{4}\right)_{2} \mathrm{SO}_{4}, 3 \mathrm{~g} \cdot \mathrm{L}^{-1} \mathrm{KH}_{2} \mathrm{PO}_{4}, 2.25 \mathrm{~g} \cdot \mathrm{L}^{-1} \mathrm{MgSO}_{4} \cdot 7 \mathrm{H}_{2} \mathrm{O}$, $33 \mu \mathrm{g} \cdot \mathrm{L}^{-1}$ biotin, and $120 \mathrm{ppm}$ Vitahop (BetaTec). The molasses (Nordic Sugar A/S) contained 40 wt \% fermentable sugars (sucrose, fructose, and glucose), lactic acid $\left(0.034 \mathrm{~g} \cdot \mathrm{g}^{-1}\right)$, and acetic acid $\left(0.008 \mathrm{~g} \cdot \mathrm{g}^{-1}\right)$. The cultivation was initiated by inoculation with the preculture. The batch cultivation was performed with $0.5 \mathrm{~L}$ working volume, with a constant aeration rate of $1 \mathrm{vvm}, \mathrm{pH}$ maintained at 5.2 and an agitation rate of $700 \mathrm{rpm}$. The batch phase was concluded when all sugars were consumed, as indicated by the evolution of carbon dioxide and oxygen in the bioreactor gas effluent.

The fed-batch phase was initiated after fermentable sugars were depleted in the batch phase. The feed solution comprised diluted wheat straw hydrolyzate liquor that was supplemented with $150 \mathrm{~g} \cdot \mathrm{L}^{-1}$ of molasses. The hydrolyzate liquor in the feed solution brought about inhibitor concentrations in the final working volume that corresponded to the concentrations in a broth with a $7.5 \mathrm{wt} \%$ WIS load. The purpose of the hydrolyzate liquor in the fed-batch phase was to improve yeast tolerance by short-term adaptation of the cultivated yeast to the environmental conditions in the fermentation experiments, per Nielsen et al. [29]. The feed solution was pulse-fed to the bioreactor at a constant rate for $20 \mathrm{~h}$ to a final working volume of $1.5 \mathrm{~L}$. The reactor was aerated by sparging at a constant rate of $1 \mathrm{vvm}$, based on the final volume, and the $\mathrm{pH}$ was maintained at 5.2 automatically with sterile $2.5 \mathrm{M} \mathrm{NaOH}$ solution.

The propagated yeast was harvested by centrifugation $(3800 \times g, 10 \mathrm{~min})$ and washed with $9 \mathrm{~g} \cdot \mathrm{L}^{-1}$ sterile $\mathrm{NaCl}$ solution. The cell pellets were resuspended in sterile $9 \mathrm{~g} \cdot \mathrm{L}^{-1} \mathrm{NaCl}$ solution to yield inocula with a cell dry matter concentration of $120 \mathrm{~g} \cdot \mathrm{L}^{-1}$.

\section{Enzymatic hydrolysis of the solid fractions}

The unwashed solid fractions from the filtered slurries of pretreated wheat straw were hydrolyzed enzymatically prior to the cofermentation (Fig. 1) in a Terrafors rotating drum reactor (Infors AG) that was agitated by free-fall mixing, yielding a glucose-rich enzymatic hydrolyzate. The unwashed solid fractions of the severe and mild slurries, containing both solids and hydrolyzate liquor, were diluted with distilled water. The dilution of the solid fraction of the severe slurry yielded a WIS load of 20 wt \% in the enzymatic hydrolysis, and the dilution of the solid fractions of the mild slurry yielded WIS loads of 26 and $32 \mathrm{wt} \%$ in the enzymatic hydrolyzes, dependent on the WIS content in the original slurry. The dilution was constrained by the target $10 \mathrm{wt} \%$ WIS in the SHCF experiments. Hydrolysis was performed at $45^{\circ} \mathrm{C}$ for $96 \mathrm{~h}$ with an enzyme load of $9 \mathrm{FPU} \cdot \mathrm{g}^{-1}$ WIS and constant 


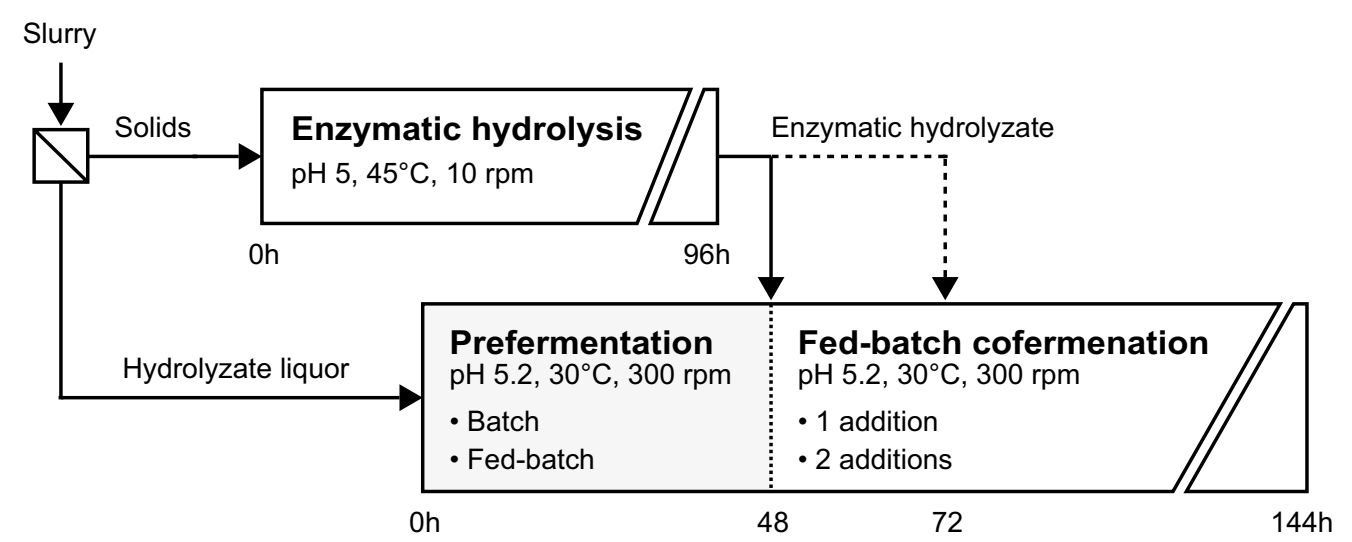

Fig. 1 Schematic of SHCF strategies

reactor revolution at $10 \mathrm{rpm}$. Cellic CTec2 enzyme preparation (Novozymes A/S) with a filter paper activity of $98 \mathrm{FPU} \cdot \mathrm{g}^{-1}$, as determined per Adney and Baker [30], was dispersed in the dilution-water to promote even distribution of enzymes. The $\mathrm{pH}$ was maintained at 5 by manual addition of $5 \mathrm{M}$ sterile $\mathrm{NaOH}$ solution.

\section{Separate hydrolysis and cofermentation}

All cofermentations were performed in sterilized $2 \mathrm{~L}$ Labfors bioreactors (Infors AG) equipped with an anchor impeller and a pitched six-blade turbine. The reactor diameter to impeller diameter ratio was 1.5 for the anchor impeller and 1.7 for the pitched six-blade turbine, and the reactor height to diameter ratio was 1.7. All cofermentations were supplemented with $0.5 \mathrm{~g} \cdot \mathrm{L}^{-1}$ $\left(\mathrm{NH}_{4}\right)_{2} \mathrm{HPO}_{4}, 0.125 \mathrm{~mL} \cdot \mathrm{L}^{-1}$ Vitahop (BetaTec), and $0.4 \mathrm{~mL} \cdot \mathrm{L}^{-1}$ Antifoam RD Emulsion (Dow Corning), all based on the final volume. The $\mathrm{pH}$ in the fermentation broths were maintained at 5.2 by automatic addition of sterile $2.5 \mathrm{M} \mathrm{NaOH}$ solution.

\section{SHCF with prefermentation}

The prefermentation of hydrolyzate liquor followed by fed-batch cofermentation of enzymatic hydrolyzate was performed with a final working weight of $1.5 \mathrm{~kg}$; a total WIS load of 10 wt \%, based on the final weight; a yeast load of $5 \mathrm{~g} \cdot \mathrm{L}^{-1}$ of dry matter (DM), based on the final volume; and an overall enzyme load of 10 FPU.g ${ }^{-1}$ WIS.

The fermentations were performed sequentially by prefermentation of the hydrolyzate liquor, followed by cofermentation of the enzymatic hydrolyzate (Fig. 1). The prefermentation of the hydrolyzate liquor was performed using either of two feed strategies: batch or fedbatch. In batch prefermentation, the entire amount of separated hydrolyzate liquor was supplied at outset and cofermented for $48 \mathrm{~h}$. For fed-batch prefermentation, a two-step process was adopted. Approximately 25 wt \% of the separated hydrolyzate liquor was cofermented in batch for $12 \mathrm{~h}$, and the remaining $75 \mathrm{wt} \%$ was fed linearly to the fermentor for the subsequent $36 \mathrm{~h}$. The amount of hydrolyzate liquor at outset was set to surpass minimum required liquid level in the fermentor $(\geq 200 \mathrm{~mL})$ to ensure reliable $\mathrm{pH}$ and temperature control. Regardless of mode, the full yeast load, $5 \mathrm{~g} \cdot \mathrm{L}^{-1}$, was pitched at outset and 1 FPU. ${ }^{-1}$ WIS of Cellic CTec2, based on total ingoing WIS, was added after $4 \mathrm{~h}$ to hydrolyze solubilized oligosaccharides. The hydrolyzate liquor was cofermented under anaerobic conditions at $30^{\circ} \mathrm{C}$ for $48 \mathrm{~h}$, and the bioreactor was agitated at $300 \mathrm{rpm}$.

Two feed strategies were used for the following fedbatch cofermentation of the enzymatic hydrolyzate: (i) all enzymatic hydrolysate was added after $48 \mathrm{~h}$, and (ii) half of the enzymatic hydrolyzate was added after $48 \mathrm{~h}$, and the remaining half added after $72 \mathrm{~h}$. Enzymes, equivalent to a total enzymatic activity of $9 \mathrm{FPU} \cdot \mathrm{g}^{-1} \mathrm{WIS}$, were carried over with the addition of enzymatic hydrolyzate, resulting in total cellulolytic activity of $10 \mathrm{FPU} \cdot \mathrm{g}^{-1} \mathrm{WIS}$, based on the total ingoing WIS content. The temperature was maintained at $30{ }^{\circ} \mathrm{C}$, and agitation was maintained at $300 \mathrm{rpm}$. The experiments were terminated after a total fermentation time of $144 \mathrm{~h}$.

\section{Model fermentations}

Model cofermentations were performed with a final working weight of $\sim 1.4 \mathrm{~kg}$ using mild and severe hydrolyzate liquor from the pretreated wheat straw slurries. The hydrolyzate liquor was diluted with distilled water to inhibitor and sugar concentrations that corresponded to a WIS load of $10 \mathrm{wt} \%$. Further, the hydrolyzate liquor was supplemented with glucose, corresponding to $81 \%$ yield in enzymatic hydrolysis of the solid fraction. The supplemented hydrolyzate liquor mimicked the composition 
of enzymatically hydrolyzed slurry. An enzyme load of 2 FPU.g ${ }^{-1}$ WIS was applied to hydrolyze solubilized oligosaccharides, and the fermentor was inoculated with a yeast load of $5 \mathrm{~g} \cdot \mathrm{L}^{-1} \mathrm{DM}$. All components were added at the outset. The model cofermentation mimicked a batch SHCF, but avoided the bias from influence of solid material and hydrolysis limitations in the cofermentation. The hydrolyzate liquor was cofermented under anaerobic conditions at $30{ }^{\circ} \mathrm{C}$ for $144 \mathrm{~h}$, and the bioreactor was agitated at $300 \mathrm{rpm}$.

\section{Analytical procedures}

Extracellular metabolites, inhibitors, and sugars were measured by high-performance liquid chromatography (HPLC) on a Shimadzu HPLC system that was equipped with an RID-10A refractive index detector (Shimadzu). Samples for carbohydrate analysis with low $\mathrm{pH}$ (from hydrolyzate liquors) were adjusted to $\mathrm{pH} 5$ with $\mathrm{CaCO}_{3}$ and centrifuged in $10 \mathrm{~mL}$ tubes $(960 \times g, 5 \mathrm{~min})$. All samples were centrifuged $(16,000 \times g, 3 \mathrm{~min})$, and the supernatants filtered through $0.20 \mu \mathrm{m}$ syringe filters (GVS Filter Technology). Filtered samples were stored at $-20^{\circ} \mathrm{C}$ until analysis.

Extracellular metabolites, organic acids, and degradation products in the hydrolyzate liquors and fermentation broth were analyzed by isocratic ion-exchange chromatography using an Aminex HPX-87H column (Bio-Rad Laboratories) at $50{ }^{\circ} \mathrm{C}$. The eluent was $5 \mathrm{mM}$ $\mathrm{H}_{2} \mathrm{SO}_{4}$, applied at a flow rate of $0.5 \mathrm{~mL} \cdot \mathrm{min}^{-1}$. Sugars and xylitol in the hydrolyzate liquors and fermentation broth were quantified by isocratic ion-exchange chromatography on an Aminex HPX-87P column (Bio-Rad Laboratories) at $85^{\circ} \mathrm{C}$. Deionized water was used as the eluent at a flow rate of $0.5 \mathrm{~mL} \cdot \mathrm{min}^{-1}$.

Dry matter content (DM) of solids and water-insoluble solids (WIS) were measured per Sluiter et al. [31], and Sluiter et al. [32], respectively. Soluble carbohydrates, monomeric sugars that were released into solution during pretreatment, and pretreatment degradation products were quantified by acid hydrolysis and HPLC per Sluiter et al. [33]. Further, structural carbohydrates, lignin, and ash contents of the water-insoluble fraction of the wheat straw slurries were measured per Sluiter et al. [34].

\section{Calculation of yields}

Ethanol yields were calculated at two levels: an overall yield and a metabolic yield. The overall ethanol yield was based on total supplied glucose and xylose; i.e. the sum of glucose and xylose present in the slurries, including monomers, oligomers, and polymers. The metabolic ethanol yield was based on consumed glucose and xylose. The mass of glucose and xylose available in the form of polymers in the WIS were corrected with factors 1.111 and 1.136, respectively, to account for the addition of water to the monomeric units during hydrolysis. The percentage of maximum theoretical ethanol yield was based on a theoretical stoichiometric yield of $0.51 \mathrm{~g} \cdot \mathrm{g}^{-1}$ on glucose and xylose.

\section{Results and discussion \\ Material preprocessing and composition}

Wheat straw was pretreated using dilute acid-catalyzed steam pretreatment at two conditions to yield two slurries denoted mild and severe slurry, based on the relative inhibitor concentrations in the hydrolyzate liquor. The lower inhibitor concentrations in the mild slurry was attributed to lower degradation, due to the shorter holdup time, and also to some extent to higher dilution in the pretreatment. The severe slurry had a water-insoluble solids (WIS) content of 13.9 wt \%, versus 11.1 to $12.7 \mathrm{wt} \%$ for the mild slurry. The variation between barrels of the mild slurry was likely due to sedimentation in the storage vessel at the demonstration-scale pretreatment site. The mild slurry with WIS content of $12.7 \mathrm{wt} \%$ was used in the evaluated fermentations of mild slurry. The mild slurry with lower WIS content (11.1 wt \%) was used to illustrate the limitations with the proposed cofermentation strategy. The applied pretreatments solubilized mainly hemicellulosic sugars. Consequently, hydrolyzate liquors that were rich in xylose and hemicellulosic oligomers, and cellulose-rich solids were obtained. The composition of the WIS fractions and the sugar and inhibitor concentrations of the hydrolyzate liquors are listed in Table 1.

The separation of hydrolyzate liquors from the lignocellulosic solids by filtration, in combination with the compositional differences between the phases (Table 1), enabled the sequential targeting of xylose and glucose conversion. The hydrolyzate liquor contained most of the xylose and inhibitors and the unwashed lignocellulosic solids harbored mainly cellulose and lignin. This allowed the use of feeding schemes that mitigate the impact of inhibitors and effect more favorable glucose-to-xylose ratios for xylose utilization could be implemented.

\section{Enzymatic hydrolysis of the solid fractions}

The enzymatic hydrolysis of the retained solids after filtration was performed at high WIS loads, because of the separation of hydrolyzate liquor from the lignocellulosic solids. The dilution of the solid fractions was further constrained by the intended WIS load of $10 \mathrm{wt} \%$ in the SHCF experiments and the aim to maximize separation between the bulk fractions of glucose and xylose.

The unwashed solid fraction of the severe slurry was diluted from a WIS content of $34 \mathrm{wt} \%$ in the retained solids to the $20 \mathrm{wt} \%$ used in the enzymatic hydrolysis. 
Table 1 Composition of structural carbohydrates and lignin in the water-insoluble fractions of the pretreated materials and sugar compositions and prevalence of inhibitory compounds in hydrolyzate liquors

\begin{tabular}{lll}
\hline & $\begin{array}{l}\text { Severe wheat straw } \\
\text { slurry }\end{array}$ & $\begin{array}{l}\text { Mild wheat straw } \\
\text { slurry }\end{array}$ \\
\hline Steam-pretreated & material (\% of dry weight) & \\
Glucan & 51.4 & 50.7 \\
Xylan & 1.7 & 1.0 \\
Galactan & 0.0 & $B D L$ \\
Arabinan & 0.1 & 0.05 \\
Mannan & 0.1 & 0.07 \\
Acid-soluble lignin & 0.7 & 0.6 \\
Acid-insoluble & 33.8 & 29.8 \\
lignin & & \\
Lignin ash & 6.9 & 10.2 \\
Total determined & 94.6 & 92.4 \\
Hydrolyzate liquor $(g \cdot L-1)$ & \\
Glucose & 14.5 & 8.7 \\
Xylose & 32.6 & 35.4 \\
Galactose & $B D L$ & 0.9 \\
Arabinose & 3.5 & 1.8 \\
Mannose & 1.8 & 0.7 \\
Formic acid & 1.6 & 0.8 \\
Acetic acid & 8.5 & 5.6 \\
Levulinic acid & $B D L$ & 0.5 \\
HMF & 1.3 & 0.3 \\
Furfural & 7.7 & 3.7 \\
\hline
\end{tabular}

$B D L$ below detection limit

The unwashed retained solids from the mild slurry was diluted from a WIS content of 44 to the 26 wt \% WIS applied in the enzymatic hydrolysis. Sufficient water for dilution was available to provide even distribution of enzyme preparation and sufficient mixing. Liquefaction became apparent after a few hours, and an extensive degradation of solids occurred during the enzymatic hydrolysis. The enzymatic hydrolysis was performed to produce a glucose-rich enzymatic hydrolysate, and not evaluated further.

Performing the enzymatic hydrolyses at high WIS loads imply the risk of lower sugar yields in hydrolysis. It has been shown for several lignocellulosic substrates that greater substrate loads decrease the corresponding hydrolysis yield [35]. The effect has been attributed to product inhibition [36,37], inhibition by sugar-derived inhibitors and lignin [38, 39], and mass transfer limitations and other effects that are related to the increased WIS loads [40]. However, inhibition primarily affects the hydrolysis rate - not the maximum conversion or yieldgiven sufficient time. Minor inefficiencies in the hydrolysis could be rectified with the employed cofermentation strategy, because the enzymes were carried over from the enzymatic hydrolysis to the subsequent cofermentation step, and provided an additional period of hydrolysis. Despite the possibility to partially rectify hydrolysis inefficiencies obtaining high sugar yields in the hydrolysis was important to successfully carry out the devised SHCF-design.

\section{Strategies for separate hydrolysis and cofermentation}

In the first cofermentation step, the prefermentation, xylose conversion was targeted. The prefermentation of the hydrolyzate liquors separated from mild and severe slurry were conducted in batch and fed-batch. In the second cofermentation step, the fed-batch cofermentation, glucose conversion was targeted, and remaining xylose coconsumed. The enzymatic hydrolyzate obtained from the enzymatic hydrolysis of the solid fractions, which contained high amounts of glucose, was fed to the prefermented hydrolyzate liquor. One or two additions of enzymatic hydrolyzate were investigated. The aim was to elucidate the impact of number of additions of enzymatic hydrolyzate, independently and in combination with different prefermentation configurations, on xylose utilization and ethanol yield.

\section{Xylose utilization in batch prefermentation}

Batch prefermentation of the separated mild hydrolyzate liquor resulted in depletion of glucose and consumption of $88 \%$ of the xylose. The deviation from the mean in the experiments was less than $1 \%$. The consumed xylose equaled $69 \%$ of the total available xylose in the used slurry. Batch prefermentation of separated mild hydrolyzate liquor elicited higher xylose utilization than with severe hydrolyzate liquor. The glucose in the severe hydrolyzate liquor was depleted and $77 \%$ of the available xylose in the hydrolyzate was consumed, which equaled $48 \%$ of the total available xylose in the used slurry. The substantial consumption of xylose during batch prefermentation reduced the extent of xylose coconsumption needed after the addition of enzymatic hydrolyzate.

The addition of enzymes after the depletion of measured glucose in the fermentation broth was intended to supply low amounts of monomeric glucose from solubilized oligomers to promote coconsumption and facilitate xylose uptake. However, declining xylose uptake rates were seen in the batch prefermentations of mild and severe hydrolyzate liquors after depletion of measured glucose (Figs. 2a, c, 3a), indicating that not enough glucose was available in the solubilized oligomers to sustain coconsumption and facilitate xylose utilization.

Batch prefermentation of mild and severe hydrolyzate liquor elicited metabolic ethanol yields of 0.34 and $0.33 \mathrm{~g} \cdot \mathrm{g}^{-1}$, respectively. The higher ethanol 

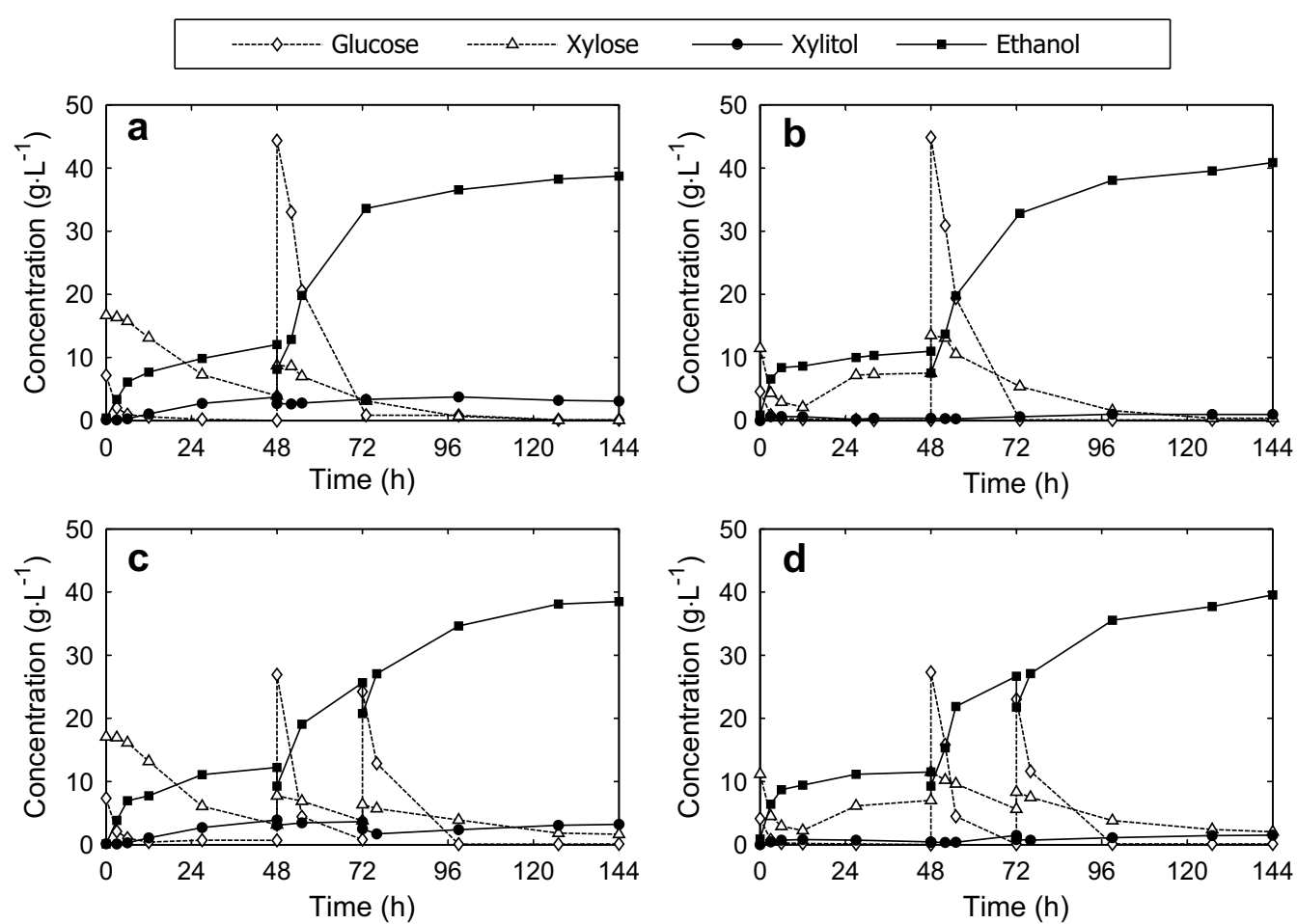

Fig. 2 Time courses for SHCF of mild wheat straw slurry with $48 \mathrm{~h}$ of (a) batch or (b) fed-batch prefermentation followed by one addition of enzymatic hydrolyzate and $48 \mathrm{~h}$ of (c) batch or (d) fed-batch prefermentation followed by two additions of enzymatic hydrolyzate
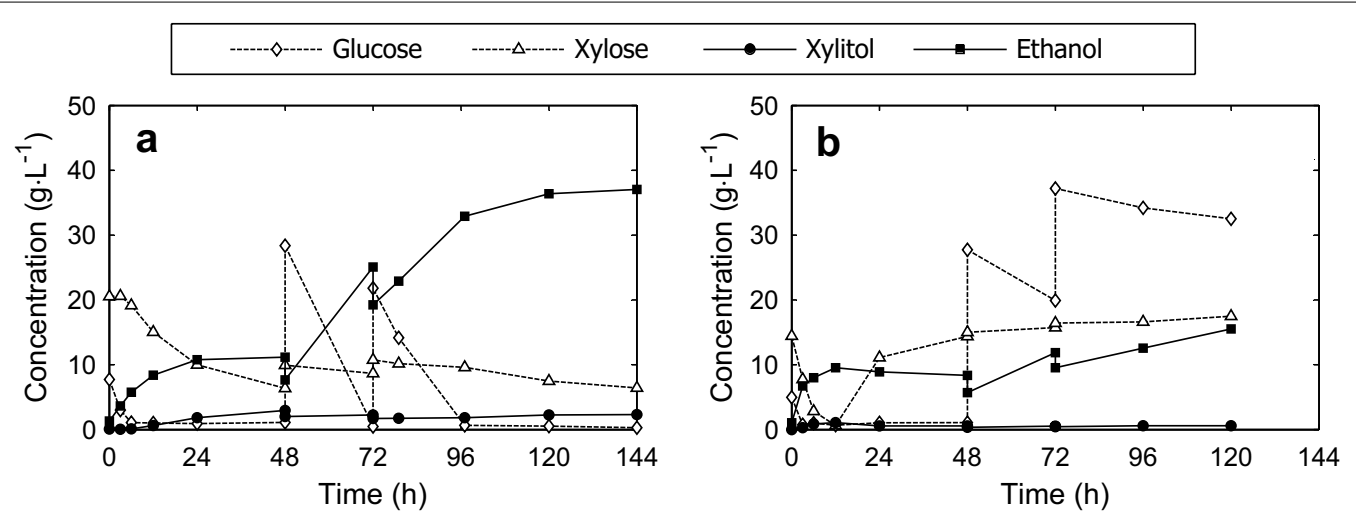

Fig. 3 Time courses for SHCF of severe wheat straw slurry with 48 h of (a) batch or (b) fed-batch prefermentation followed by two additions of enzymatic hydrolysate

concentrations and metabolic ethanol yields obtained with mild hydrolyzate liquor were attributed to the higher xylose utilization (Table 2) and the lower inhibitor concentrations (Table 1), respectively.

\section{Xylose utilization in fed-batch prefermentation}

To address the inability to sustain coconsumption of xylose and glucose by liberating glucose from the solubilized oligomers in the hydrolyzate liquors, the fed-batch prefermentation strategy was implemented. The fedbatch layout was intended to continuously supply low amounts of glucose and promote coconsumption. The glucose-to-xylose ratio of the hydrolyzate liquors was such (Table 1) that a low measured glucose concentration in the bioreactor could be maintained with a feed of hydrolyzate liquor (Figs. 2b, d, 3b), which kinetically favored xylose consumption [17]. However, lower xylose utilization was observed with fed-batch prefermentation, 
Table 2 Summary of prefermentation results

\begin{tabular}{|c|c|c|c|c|}
\hline & \multicolumn{2}{|c|}{$\begin{array}{l}\text { Severe wheat straw } \\
\text { slurry }\end{array}$} & \multicolumn{2}{|c|}{$\begin{array}{l}\text { Mild wheat straw } \\
\text { slurry }\end{array}$} \\
\hline & Batch & Fed-batch & Batch & Fed-batch \\
\hline \multicolumn{5}{|c|}{ Residual sugars and end-products $\left[\mathrm{g} \cdot \mathrm{L}^{-1}\right]$} \\
\hline Glucose & 0.1 & 0.1 & 0.0 & 0.1 \\
\hline Xylose & 6.4 & 14.4 & 3.7 & 7.3 \\
\hline Ethanol & 11.2 & 8.4 & 12.1 & 11.2 \\
\hline Xylitol & 2.9 & 0.6 & 3.8 & 0.4 \\
\hline $\begin{array}{l}\text { Metabolic ethanol yield } \\
{\left[\mathrm{g} \cdot \mathrm{g}^{-1}\right]^{a}}\end{array}$ & 0.329 & 0.322 & 0.342 & 0.353 \\
\hline$\%$ of theoretical & 64.4 & 63.1 & 67.1 & 69.2 \\
\hline Xylose utilization [\%] & 77.3 & 48.6 & 88.2 & 76.9 \\
\hline Xylitol yield $\left[\mathrm{g} \cdot \mathrm{g}^{-1}\right]^{\mathrm{b}}$ & 0.136 & 0.041 & 0.137 & 0.017 \\
\hline
\end{tabular}

as compared with batch, with both hydrolyzate liquors (Table 2). On average 77 and $49 \%$ of xylose available in the mild and severe hydrolyzate liquor, respectively, was consumed; as compared to 88 and $77 \%$, respectively, in batch. The deviation from the mean in fed-batch prefermentation experiments was less than $3 \%$. The underlying reason for the lower xylose consumption was that the feed rate of xylose-rich hydrolyzate liquor exceeded the xylose uptake rate. In combination with preferential consumption of glucose, this resulted in the accumulation of xylose during the fed-batch phase of the prefermentation (Figs. 2b, d, 3b). Although lower xylose utilizations were achieved with fed-batch prefermentation, substantial fractions of total available xylose in the slurries were consumed. The consumed xylose in mild and severe hydrolyzate liquor after fed-batch prefermentation corresponded to 60 and $31 \%$, respectively, of total available xylose.

The lower xylose consumptions in fed-batch prefermentation were mirrored in the obtained ethanol concentrations (Table 2), but did not have a significant effect on the metabolic ethanol yields (Table 2). Metabolic ethanol yields of 0.353 and $0.322 \mathrm{~g} \cdot \mathrm{g}^{-1}$ were obtained with fed-batch prefermentation of mild and severe hydrolyzate liquor, respectively, as compared to 0.342 and $0.329 \mathrm{~g} \cdot \mathrm{g}^{-1}$ with batch prefermentation. The deviations from the mean metabolic ethanol yields were less than $2 \%$ in all instances. Fed-batch prefermentations were also accompanied with lower xylitol production than batch prefermentation. Batch prefermentation of mild and severe hydrolyzate liquor resulted in xylitol yields of 0.136 and $0.134 \mathrm{~g} \cdot \mathrm{g}^{-1}$, respectively, whereas only $0.017 \mathrm{~g} \cdot \mathrm{g}^{-1}$ respectively $0.041 \mathrm{~g} \cdot \mathrm{g}^{-1}$ were produced in fed-batch prefermentation. The deviations from the mean in batch prefermentation experiments were less than $2 \%$, and less than $7 \%$ in fed-batch prefermentation experiments. The difference in xylitol production between the prefermentation configurations was attributed to the higher xylose consumption rate in batch prefermentation. High xylose consumption rates create a metabolic bottleneck because of an imbalance of cofactors in engineered XR/XDHpathways [11, 12], whereas it has been shown that lower consumption rates suppress xylitol production [41]. The lower xylose consumption in fed-batch prefermentation in combination with continuous availability of low concentrations of furaldehydes, which act as external electron sinks for the regeneration of cofactors [42], likely alleviated the cofactor imbalance.

\section{Effect of prefermentation on overall xylose utilization}

The selected feed strategy during prefermentation had several intertwined consequences on fermentation performance during the subsequent fed-batch cofermentation of enzymatic hydrolyzate. The different prefermentation configurations had different impacts on fermentative capacity of the yeast during the fed-batch cofermentation of enzymatic hydrolyzate, which was primarily attributed to changes in the viability.

Comparison of overall cofermentation outcome between batch and fed-batch prefermentation of severe hydrolyzate liquor followed by two additions of enzymatic hydrolyzate (Fig. 3) exemplify the different consequences in a high inhibitor concentration context. Batch prefermentation followed by fed-batch cofermentation of enzymatic hydrolyzate elicited a xylose utilization of $71 \%$ and an overall ethanol yield of $0.381 \mathrm{~g} \cdot \mathrm{g}^{-1}$. In contrast, the corresponding strategy with fed-batch prefermentation resulted in low glucose conversion and, seemingly, no xylose consumption after fed-batch prefermentation of severe hydrolyzate liquor (Fig. 3b). Fed-batch prefermentation supplied lower initial concentrations of inhibitors than batch prefermentation, but the continuous feed of hydrolyzate liquor seemingly exhausted the yeast. The continuous feed of inhibitors during fed-batch prefermentation exceeded the detoxification rate, which resulted in the accumulation of non-metabolized furaldehydes (data not shown). The furaldehydes were not fully converted during the sequent fed-batch cofermentation of enzymatic hydrolyzate. In contrast, the yeast was resilient to the higher initial inhibitor concentrations in batch prefermentation. The yeast was capable of promptly detoxifying the hydrolyzate liquor during batch prefermentation and after each addition of enzymatic hydrolyzate (data not shown). The decreased viability and fermentative capacity of the yeast was likely due to ceased growth and inhibitory effects [3]. The yeast cells 
were assumed to be particularly sensitive to exposure to inhibitors during the prefermentation of xylose-rich hydrolyzate liquor, because the conversion of xylose does not support growth efficiently [43].

When hydrolyzate liquor and enzymatic hydrolyzate from mild slurry was utilized, the xylose fermenting capacity was sustained throughout $144 \mathrm{~h}$ of cofermentation. However, lower xylose utilization was obtained after fed-batch cofermentation of enzymatic hydrolyzate when fed-batch prefermentation was applied, as compared to the corresponding strategy with batch prefermentation (Table 3). Fed-batch prefermentation with one or two additions of enzymatic hydrolyzate elicited xylose utilizations of 98 and $91 \%$, respectively; whereas the corresponding cofermentations with batch prefermentation elicited xylose utilizations of 97 and $93 \%$, respectively. The deviations from the means for experiments employing fed-batch prefermentation was less than $3 \%$. The primary reason was higher residual xylose concentrations at the end of fed-batch cofermentations of enzymatic hydrolysate when fed-batch prefermentation was applied (Table 3). This suggests that fed-batch prefermentation negatively influences the xylose fermenting capacity.

\section{Effect of prefermentation on overall xylitol production}

Strategies employing fed-batch prefermentations elicited lower xylitol yields than those with batch prefermentation (Table 3). The xylitol production was decreased with fed-batch prefermentation, regardless of whether mild or severe slurry was used, and effected lower overall xylitol yields at the end of the fed-batch cofermentation of enzymatic hydrolyzate (Table 3 ). Because of the sequential targeting of xylose and glucose conversion in the fermentation steps, the xylitol productions during prefermentation were major determinants of total xylitol production. After prefermentation little xylitol was produced, because glucose was the predominant substrate and remaining xylose was mainly coconsumed.

For all practical purposes xylitol production represents a loss of carbon that could be converted to ethanol. The lower xylitol production in strategies employing fedbatch prefermentation contributed to the xylose conversion efficiency and, thus, the higher overall ethanol yields obtained. Despite lower xylose utilization the overall ethanol yields were higher than for strategies employing batch prefermentation (Table 3). The higher overall ethanol yields were correlated with the decreased xylitol production obtained with fed-batch prefermentation.

\section{Effect of number of additions of enzymatic hydrolyzate}

The multiple additions of enzymatic hydrolyzate during the fed-batch cofermentation provided the means to lower glucose concentrations in the fermentation broth, as compared to a single addition. Fed-batch SHCF of steam-pretreated wheat straw has previously been shown to enhance xylose utilization [22, 23]. However, regardless of chosen prefermentation configuration a single addition of enzymatic hydrolyzate elicited higher xylose utilization and ethanol yields than corresponding fedbatch cofermentations with two additions (Table 3). The yeast exhibited decreased xylose fermenting capacity with repetitive addition of mild enzymatic hydrolyzate

Table 3 Summary of results after prefermentation of hydrolyzate liquor followed by fed-batch cofermentation of enzymatic hydrolyzate

\begin{tabular}{|c|c|c|c|c|c|c|c|c|c|}
\hline \multirow[t]{3}{*}{ Fig. } & \multirow[t]{3}{*}{ Description } & \multicolumn{4}{|c|}{ Residual sugars and end-products } & \multirow{3}{*}{$\begin{array}{l}\text { Xylose utilization } \\
\%\end{array}$} & \multicolumn{3}{|l|}{ Yields } \\
\hline & & \multirow{2}{*}{$\begin{array}{l}\text { Glucose } \\
\mathrm{g} \cdot \mathrm{L}^{-1}\end{array}$} & \multirow{2}{*}{$\begin{array}{l}\text { Xylose } \\
g \cdot L^{-1}\end{array}$} & \multirow{2}{*}{$\begin{array}{l}\text { Xylitol } \\
g \cdot L^{-1}\end{array}$} & \multirow{2}{*}{$\begin{array}{l}\text { Ethanol } \\
\mathrm{g} \cdot \mathrm{L}^{-1}\end{array}$} & & \multicolumn{2}{|c|}{ Ethanol $^{\mathrm{a}}$} & \multirow{2}{*}{$\begin{array}{l}\text { Xylitol }^{\mathbf{b}} \\
\mathbf{g} \cdot \mathbf{g}^{-1}\end{array}$} \\
\hline & & & & & & & $g \cdot g^{-1}$ & $\%$ & \\
\hline & S, Model & 0.1 & 5.3 & 2.0 & 28.9 & 75.9 & 0.346 & 67.9 & 0.120 \\
\hline & M, Model & 0.1 & 1.2 & 5.7 & 32.2 & 96.1 & 0.360 & 70.6 & 0.191 \\
\hline $2 \mathrm{a}$ & $S, B, 2$ & 0.3 & 6.4 & 2.3 & 37.0 & 70.6 & 0.381 & 74.8 & 0.135 \\
\hline $2 \mathrm{~b}$ & $S, F B, 2$ & 32.5 & 17.5 & 0.6 & 16.0 & $N D$ & 0.164 & 32.2 & $N D$ \\
\hline $3 a$ & $M, B, 1$ & 0.1 & 0.1 & 3.1 & 38.7 & 98.0 & 0.401 & 78.5 & 0.112 \\
\hline $3 b$ & $M, F B, 1$ & 0.1 & 0.4 & 1.0 & 40.9 & 96.9 & 0.423 & 82.9 & 0.036 \\
\hline $3 c, 4 b$ & $M, B, 2$ & 0.1 & 1.6 & 3.2 & 38.5 & 92.4 & 0.398 & 78.1 & 0.123 \\
\hline $4 a$ & $M, B, 2^{C}$ & 0.5 & 4.7 & 1.4 & 27.3 & $N D$ & 0.287 & 56.2 & $N D$ \\
\hline $3 d$ & $M, F B, 2$ & 0.1 & 2.0 & 1.5 & 39.6 & 90.9 & 0.409 & 80.2 & 0.058 \\
\hline
\end{tabular}

S severe slurry; $M$ mild slurry; $B$ batch prefermentation; $F B$ fed-batch prefermentation; 11 addition of enzymatic hydrolyzate; 22 additions of enzymatic hydrolyzate, ND not determined

a Ethanol yield based on total supplied glucose and xylose and related to the maximum theoretical yield $\left(0.51 \mathrm{~g} \cdot \mathrm{g}^{-1}\right)$

b Xylitol yield based on total consumed xylose

c $12, M, B, 2$ with insufficient prehydrolysis 
that contained inhibitors. The trend was evidenced by higher final residual xylose concentrations (Table 3).

Because xylose is converted at lower rates than glucose [8], the fermentation time becomes of essence. The distributed multiple additions of enzymatic hydrolyzate effectively reduce the average time available for conversion of xylose that resides in the enzymatic hydrolyzate. Another aspect relates to the addition of inhibitors. The measured inhibitors were predominantly present in the separated hydrolyzate liquors, and were to a large extent converted to less toxic entities during the prefermentation. However, contributions of inhibitors were made with every addition of enzymatic hydrolyzate, because 20-30 wt \% of the total amount of hydrolyzate liquor in the slurry after pretreatment remain with the solids after the filtration. In the experiment series with mild slurry the yeast was able to convert furfural irrespective of prefermentation mode and number of additions of enzymatic hydrolyzate. HMF, on the other hand, was only fully converted with batch prefermentation and one addition of enzymatic hydrolyzate (data not shown). Fed-batch prefermentation decreased the conversion of HMF, and in combination with two additions of enzymatic hydrolyzate no HMF was converted after prefermentation (data not shown). The trend in furaldehyde conversion was correlated to that of the xylose fermenting capacity. The aggregated effect of fed-batch prefermentation and multiple additions of enzymatic hydrolysate elicited increasingly higher residual xylose concentrations (Table 3), and thus lower xylose utilization. This finding was indicative of that decreases in viability of the yeast occurred with both continuous and repetitive addition of substrate that contained inhibitors.

\section{Maximization of ethanol yield}

The highest overall ethanol yield was obtained with low concentrations of inhibitor in the slurry, because it permitted feeding schemes that maximized xylose conversion efficacy while the viability of the fermenting microorganism was sustained. The best results were obtained with fed-batch prefermentation and a single addition of enzymatic hydrolyzate, with an overall ethanol yield of $0.423 \mathrm{~g} \cdot \mathrm{g}^{-1}$, xylose utilization of $98 \%$, and the lowest xylitol production at $0.036 \mathrm{~g} \cdot \mathrm{g}^{-1}$. The deviations from the means were below $4 \%$ in all instances. The strategy combined lower xylitol productions and higher ethanol yields elicited by fed-batch prefermentation with the lower effect of inhibitors associated with one addition of enzymatic hydrolyzate. A trade-off existed between promoting efficient xylose conversion with substrate feeding and maintaining yeast viability. Ethanol yield was maximized with a balance between them.
The reduction in residual xylose concentrations and decreased xylitol production (Table 3), compared to the model fermentations, indicate that the sequential targeting of xylose and glucose conversion is a feasible way to improve xylose conversion with SHCF-based conversion strategies. The proposed strategy leaves degrees of freedom in the design to implement feeding schemes that accommodate the traits of various fermenting microorganisms, as to mitigate the inhibitory effects, sustain yeast viability and maximize xylose conversion.

\section{Method limitations}

The necessitated high WIS enzymatic hydrolysis constituted a weakness of the proposed method, because the hydrolysis outcome had profound effect on the cofermentation. The shortcoming was evidenced by the difference between cofermentations of mild slurry with an original WIS content of 11.1 and 12.7 wt \% (Table 3; Fig. 4). Batch prefermentation followed by two additions of enzymatic hydrolyzate was applied as cofermentation strategy.

The dilution of the retained solids after filtration of the mild slurry with the lower WIS content (11.1 wt \%) was severely constrained by the required WIS load of $10 \mathrm{wt} \%$ in the SHCF. Hence, the retained solids were only diluted to a WIS load of $32 \mathrm{wt} \%$ in the enzymatic hydrolysis step. The enzymatic hydrolysis resulted in inferior hydrolysis because of various reasons associated with high WIS applications [35-37]. With inferior hydrolysis the lignocellulosic solids that remained in the enzymatic hydrolyzate were partially hydrolyzed and fermented simultaneously during the fed-batch cofermentation and the benefits of the preparatory hydrolysis of the unwashed solids waned. The simultaneous hydrolysis and cofermentation was undesirable in the SHCF, because the solids were partially hydrolyzed under suboptimal conditions at $30{ }^{\circ} \mathrm{C}$ during the fed-batch cofermentation. The drawback was not necessarily a lower yield given sufficient time, but that hydrolysis rate became a limiting factor for the fermentation rate. This constrained the final ethanol yield for the limited cofermentation period. An increase in temperature during the fed-batch cofermentation of the enzymatic hydrolyzate would have been needed to accommodate hydrolysis of remaining solids and avoid severe limitations to the fermentation rate. The illustrated limitation puts emphasis on the necessity of high sugar yields in preparatory enzymatic hydrolysis to implement the sequential targeting of xylose and glucose conversion with this strategy.

\section{Conclusions}

Prefermentation of hydrolyzate liquor followed by fedbatch separate hydrolysis and cofermentation improved ethanol yields, yet batch and fed-batch prefermentation 

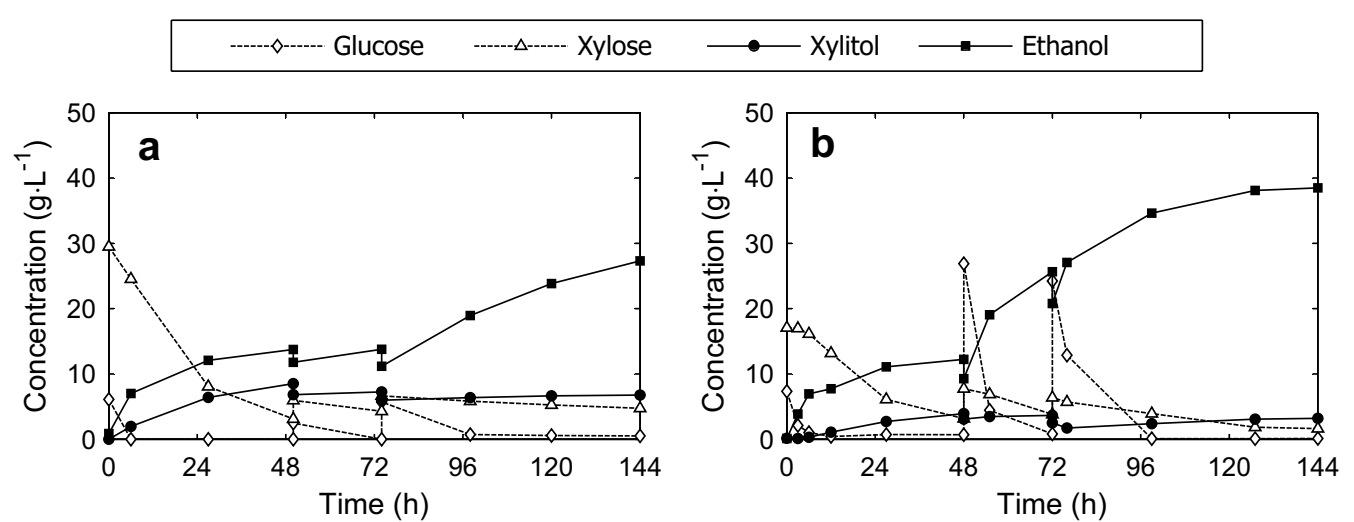

Fig. 4 Effects of insufficient prehydrolysis on fermentation behaviour. Comparison of SHCF of mild wheat straw slurry with batch prefermentation and two additions of enzymatic hydrolyzate, where (a) is with insufficiently hydrolyzed solids and $(\mathbf{b})$ is with adequate hydrolysis

had different impacts on the cofermentation. Under influence of lower inhibitor concentrations fed-batch prefermentation resulted in lower xylitol production during all steps of the fermentation and prompted higher final ethanol yields compared to corresponding cofermentations with batch prefermentation. Under influence of high inhibitor concentrations, sustained fermentation capacity was paramount to obtain improved ethanol yield. S. cerevisiae KE6-12 was resilient to high inhibitor concentrations, but succumbed to continuous exposure to inhibitors. Regardless of type of slurry, continuous feed during prefermentation of the hydrolyzate liquor and multiple additions of enzymatic hydrolyzate-and their combination-appeared to hamper the fermentative capacity and exhaust the cells. The viability of the yeast, not glucose repression of xylose metabolism, appeared to be the limiting factor for higher ethanol yields in the cofermentations. The best results were obtained with mild slurry, applying fed-batch prefermentation and a single addition of enzymatic hydrolyzate. An ethanol yield of $0.423 \mathrm{~g} \cdot \mathrm{g}^{-1}$, based on supplied glucose and xylose, and the lowest xylitol production, $0.036 \mathrm{~g} \cdot \mathrm{g}^{-1}$, was obtained. A trade-off existed between promoting xylose conversion with substrate feeding and maintaining yeast viability, and ethanol yield was maximized with a balance between them.

\footnotetext{
Abbreviations

DM: dry matter; FPU: filter paper unit; HMF: 5-hydroxymethylfurfural; HPLC: high-performance liquid chromatography; SHCF: separate hydrolysis and cofermentation; SSCF: simultaneous saccharification and cofermentation; vvm: gas volume flow per unit of liquid volume per minute; WIS: water-insoluble solids; XDH: xylitol dehydrogenase; XK: xylulokinase; XR: xylose reductase.
}

\section{Authors' contributions}

FN participated in the conception and design of the study, performed the experiments and wrote the manuscript. GZ participated in the conception and design of the study and critically reviewed the manuscript. OW and MG assisted in interpreting the data, participated in the editing and preparation of the final manuscript, and critically reviewed the manuscript. All authors read and approved the final manuscript.

\section{Acknowledgements}

The Swedish Energy Agency is gratefully acknowledged for their financial support (Process development for combined pentose and hexose fermentation, 35354-1), as is Taurus Energy AB for cofinancing the project.

\section{Competing interests}

The study was part of the project "Process development for combined pentose and hexose fermentation", which was co-financed by Taurus Energy AB. $\mathrm{GZ}$ is a shareholder and member of the board at Taurus Energy AB. Remaining authors declares no financial competing interests and all authors declare that they have no non-financial competing interests.

Received: 13 April 2016 Accepted: 7 June 2016

Published online: 01 July 2016

\section{References}

1. von Sivers M, Zacchi G (1996) Ethanol from lignocellulosics: a review of the economy. Bioresour Technol 56:131-140

2. Olsson L, Hahn-Hägerdal B (1996) Fermentation of lignocellulosic hydrolysates for ethanol production. Enzym Microb Technol 18(5):312-331

3. Palmqvist $E$, Hahn-Hägerdal B (2000) Fermentation of lignocellulosic hydrolysates. II: inhibitors and mechanisms of inhibition. Bioresour Technol 74:25-33

4. Toivari MH, Salusjärvi L, Ruohonen L, Penttilä M (2004) Endogenous Xylose Pathway in Saccharomyces cerevisiae. Appl Environ Microbiol 70(6):3681-3686

5. Kötter P, Amore R, Hollenberg CP, Ciriacy M (1990) Isolation and characterization of the Pichia stipitis xylitol dehydrogenase gene, XYL2, and construction of a xylose-utilizing Saccharomyces cerevisiae transformant. Curr Genet 18(6):493-500

6. Eliasson A, Christensson C, Wahlbom CF, Hahn-Hägerdal B (2000) Anaerobic xylose fermentation by recombinant Saccharomyces cerevisiae carrying XYL1, XYL2, and XKS1 in mineral medium chemostat cultures. Appl Environ Microbiol 66(8):3381-3386

7. van Maris AJ, Winkler AA, Kuyper M, de Laat WT, van Dijken JP, de Pronk JT (2007) Development of efficient xylose fermentation in Saccharomyces cerevisiae: xylose isomerase as a key component. Adv Biochem Eng Biotechnol 108:179-204

8. Matsushika A, Inoue H, Kodaki T, Sawayama S (2009) Ethanol production from xylose in engineered Saccharomyces cerevisiae strains: current state and perspectives. Appl Microbiol Biotechnol 84(1):37-53 
9. Toivari MH, Aristidou A, Ruohonen L, Penttilä M (2001) Conversion of xylose to ethanol by recombinant Saccharomyces cerevisiae: importance of xylulokinase (XKS1) and oxygen availability. Metab Eng 3(3):236-249

10. Kötter P, Ciriacy M (1993) Xylose fermentation by Saccharomyces cerevisiae. Appl Microbiol Biotechnol 38(6):776-783

11. Verho R, Londesborough J, Penttilä M, Richard P (2003) Engineering redox cofactor regeneration for improved pentose fermentation in Saccharomyces cerevisiae. Appl Environ Microbiol 69(10):5892-5897

12. Bruinenberg PM, Bot PH, van Dijken JP, Scheffers WA (1983) Engineering redox cofactor regeneration for improved pentose fermentation in Saccharomyces cerevisiae. Eur J Appl Microbiol Biotechnol 18:287-292

13. Watanabe S, Abu Saleh A, Pack SP, Annaluru N, Kodaki T, Makino K (2007) Ethanol production from xylose by recombinant Saccharomyces cerevisiae expressing protein-engineered NADH-preferring xylose reductase from Pichia stipitis. Microbiology 153(9):3044-3054

14. Petschacher B, Nidetzky B (2008) Altering the coenzyme preference of xylose reductase to favor utilization of NADH enhances ethanol yield from xylose in a metabolically engineered strain of Saccharomyces cerevisiae. Microb Cell Fact 7:9

15. Almeida JRM, Modig T, Petersson A, Hähn-Hägerdal B, Lidén G, GorwaGrauslund MF (2007) Increased tolerance and conversion of inhibitors in lignocellulosic hydrolysates by Saccharomyces cerevisiae. J Chem Technol Biotechnol 82(4):340-349

16. Hamacher T, Becker J, Gardonyi M, Hahn-Hägerdal B, Boles E (2002) Characterization of the xylose-transporting properties of yeast hexose transporters and their influence on xylose utilization. Microbiology 148:2783-2788

17. Meinander NQ, Boels I, Hahn-Hägerdal B (1999) Fermentation of xylose/ glucose mixtures by metabolically engineered Saccharomyces cerevisiae strains expressing XYL1 and XYL2 from Pichia stipitis with and without overexpression of TAL1. Bioresour Technol 68(1):79-87

18. Olsson L, Soerensen HR, Dam BP, Christensen H, Krogh KM, Meyer AS (2006) Separate and simultaneous enzymatic hydrolysis and fermentation of wheat hemicellulose with recombinant xylose utilizing Saccharomyces cerevisiae. Appl Biochem Biotechnol 129(1-3):117-129

19. Pitkänen J-P, Aristidou A, Salusjärvi L, Ruohonen L, Penttilä M (2003) Metabolic flux analysis of xylose metabolism in recombinant Saccharomyces cerevisiae using continuous culture. Metab Eng 5(1):16-31

20. Boles E, Müller S, Zimmermann FK (1996) A multi-layered sensory system controls yeast glycolytic gene expression. Mol Microbiol 19(3):641-642

21. Tomás-Pejó E, Oliva JM, Ballesteros M, Olsson L (2008) Comparison of SHF and SSF processes from steam-exploded wheat straw for ethanol production by xylose-fermenting and robust glucose-fermenting Saccharomyces cerevisiae strains. Biotechnol Bioeng 100(6):1122-1131

22. Erdei B, Frankó B, Galbe M, Zacchi G (2012) Separate hydrolysis and cofermentation for improved xylose utilization in integrated ethanol production from wheat meal and wheat straw. Biotechnol Biofuels 5(1):1-13

23. Erdei B, Frankó B, Galbe M, Zacchi G (2013) Glucose and xylose cofermentation of pretreated wheat straw using mutants of $S$. cerevisiae TMB3400. J Biotechnol 164(1):50-58

24. Bertilsson M, Olofsson K, Lidén G (2009) Prefermentation improves xylose utilization in simultaneous saccharification and co-fermentation of pretreated spruce. Biotechnol Biofuels 2(8):1

25. Jin M, Lau MW, Balan V, Dale BE (2010) Two-step SSCF to convert AFEXtreated switchgrass to ethanol using commercial enzymes and Saccharomyces cerevisiae 424A(LNH-ST). Bioresour Technol 101(21):8171-8178

26. Albers E, Halpin R, Olsson L (2016) Evolutionary engineering for development of improved xylose utilization capacity and inhibitor tolerance in an industrial Saccharomyces cerevisiae strain. (Manuscript in preparation)
27. Wahlbom CF, van Zyl WH, Jönsson LJ, Hahn-Hägerdal B, Otero RRC (2003) Generation of the improved recombinant xylose-utilizing Saccharomyces cerevisiae TMB 3400 by random mutagenesis and physiological comparison with Pichia stipitis CBS 6054. FEMS Yeast Res 3(3):319-326

28. Taherzadeh MJ, Lidén G, Gustafsson L, Niklasson C (1996) The effects of pantothenate deficiency and acetate addition on anaerobic batch fermentation of glucose by Saccharomyces cerevisiae. Appl Microbiol Biotechnol 46(2):176-182

29. Nielsen F, Tomás-Pejó E, Olsson L, Wallberg O (2015) Short-term adaptation during propagation improves the performance of xylose-fermenting Saccharomyces cerevisiae in simultaneous saccharification and cofermentation. Biotechnol Biofuels 8(1):1-15

30. Adney B, Baker J (1996) Measurement of cellulase activities. National Renewable Energy Laboratory, Golden

31. Sluiter A, Hames B, Hyman D, Payne C, Ruiz R, Scarlata C, Sluiter J, Templeton D, Wolfe J (2008) Determination of total solids in biomass and total dissolved solids in liquid process samples. National Renewable Energy Laboratory, Golden

32. Sluiter A, Hyman D, Payne C, Wolfe J (2008) Determination of insoluble solids in pretreated biomass material. National Renewable Energy Laboratory, Golden

33. Sluiter A, Hames B, Ruiz R, Scarlata C, Sluiter J, Templeton D, Crocker D (2006) Determination of sugars, byproducts, and degradation products in liquid fraction process samples. National Renewable Energy Laboratory, Golden

34. Sluiter A, Hames B, Ruiz R, Scarlata C, Sluiter J, Templeton D, Crocker D (2008) Determination of structural carbohydrates and lignin in biomass. National Renewable Energy Laboratory, Golden

35. Kristensen JB, Felby C, Jørgensen H (2009) Yield-determining factors in high-solids enzymatic hydrolysis of lignocellulose. Biotechnol Biofuels 2(1):11

36. Mohagheghi A, Tucker M, Grohmann K, Wyman C (1992) High solids simultaneous saccharification and fermentation of pretreated wheat straw to ethanol. Appl Biochem Biotechnol 33(2):67-81

37. Hodge DB, Karim MN, Schell DJ, McMillan JD (2008) Soluble and insoluble solids contributions to high-solids enzymatic hydrolysis of lignocellulose. Bioresour Technol 99(18):8940-8948

38. Panagiotou G, Olsson L (2007) Effect of compounds released during pretreatment of wheat straw on microbial growth and enzymatic hydrolysis rates. Biotechnol Bioeng 96(2):250-258

39. Pan $X(2008)$ Role of functional groups in lignin inhibition of enzymatic hydrolysis of cellulose to glucose. J Biobased Mater Bioenerg 2(1):25-32

40. Georgieva T, Hou X, Hilstrøm T, Ahring B (2008) Enzymatic hydrolysis and ethanol fermentation of high dry matter wet-exploded wheat straw at low enzyme loading. Appl Biochem Biotechnol 148(1-3):35-44

41. Novy V, Krahulec S, Longus K, Klimacek M, Nidetzky B (2013) Co-fermentation of hexose and pentose sugars in a spent sulfite liquor matrix with genetically modified Saccharomyces cerevisiae. Bioresour Technol 130:439-448

42. Wahlbom CF, Hahn-Hägerdal B (2002) Furfural, 5-hydroxymethyl furfural, and acetoin act as external electron acceptors during anaerobic fermentation of xylose in recombinant Saccharomyces cerevisiae. Biotechnol Bioeng 78(2):172-178

43. Sonderegger M, Jeppsson M, Larsson C, Gorwa-Grauslund M-F, Boles E, Olsson L, Spencer-Martins I, Hahn-Hägerdal B, Sauer U (2004) Fermentation performance of engineered and evolved xylose-fermenting Saccharomyces cerevisiae strains. Biotechnol Bioeng 87(1):90-98 